\title{
Foundation dentists collaborating with community pharmacies
}

\author{
By Molly Watton, DCT in Oral and Maxillofacial Surgery, Royal London Hospital, London, UK
}

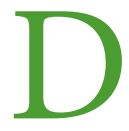

uring the COVID-19 pandemic, many healthcare professionals have been redeployed throughout the NHS, including foundation dentists (FDs).

During the first peak of the COVID-19 pandemic, the Health Education England (HEE) region of London and Kent, Surrey and Sussex provided FDs with many opportunities to volunteer for redeployment, including: clinical support within ICU units at the London Nightingale Hospital and Northwick Park Hospital, NHS 111 call handling, swabbing in social care settings, contact tracing, Pandemic Multiagency
Although oral health is gradually improving in England, in 2018, an oral health survey of adults found more than a quarter of participants (27\%) had caries, with 53\% complaining of gingival bleeding, ${ }^{1}$ and in 2019, it was estimated $23.4 \%$ of five-year-olds had caries, with an average of 3.4 teeth affected. ${ }^{2}$ With oral disease still prevalent throughout the community and with an increasingly ageing population, ${ }^{3}$ it is vital that patients can easily access dental care and advice. Community pharmacies are easily accessible and are approached frequently for oral health advice, ${ }^{4}$ hence prove to be a useful base for

\section{'Community pharmacies are easily accessible and approached frequently for oral health advice'}

Response Teams and roles within local community pharmacies.

As a result of the ongoing pandemic, the previous cohort of FDs (2019-2020) had a disrupted year with their practical learning restricted. Nevertheless, many skills were taken from redeployment opportunities where available and FDs stayed abreast of their learning with the provision of remote study days, online CPD modules, webinars and evidence-based projects, along with altered nationally agreed COVID-19 competency numbers to allow for satisfactory completion of the dental foundation training (DFT) year. But what does this mean for the current cohort of FDs?

Redeployment has allowed for consideration of cross-professional collaboration with community pharmacies to maximise FDs' exposure to patients and clinical experience. inter-professional working, which may be particularly helpful for patients with chronic non-communicable diseases such as diabetes. ${ }^{5}$ Community pharmacies have seen an increased influx of patients during COVID-19, most likely as both GPs and dental practices have been operating a restricted service, heavily reducing face-to-face consultations and predominantly relying on telephone consultations during the first peak.

The primary focus of the placement would be directed towards offering evidence-based oral health and oral hygiene advice to patients who attend community pharmacies with dental queries. Queries could include, but are not limited to: oral health advice, oral ulceration, teething, myofascial pain/TMJ pain, dry mouth, lost or fractured fillings and crowns, pericoronitis, bleeding and swollen gums, toothache and bleeding after an extraction. It would expand on the work

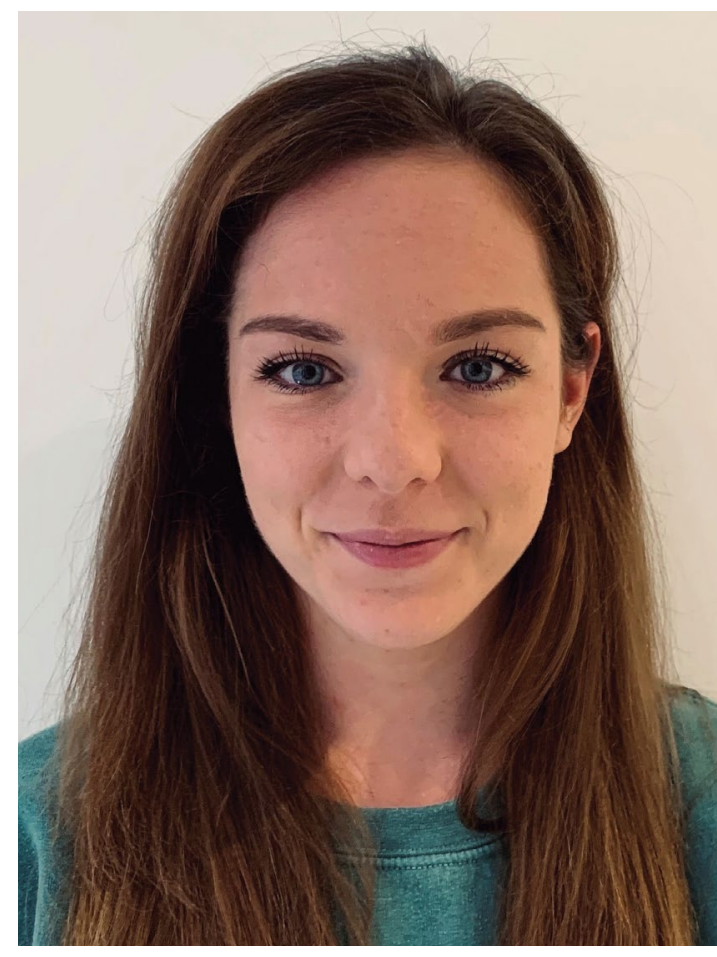

HEE Dental and Pharmacy teams in London and the South East (LaSE) introduced in 2019 , where a series of dental factsheets focusing on the main topics above were produced. These aimed to help community pharmacy teams to manage urgent dental complaints and signpost patients to other services where indicated. ${ }^{6}$

The aim of DFT is to allow for newly qualified dentists (safe beginners) to develop clinical and administrative competencies to be able to work independently, ${ }^{7}$ and to facilitate the transition from dental school into NHS general practice, which previously allowed for a broad experience to be gained. The impact of COVID-19 on the dental profession may not make this possible again until vaccination can be used to protect the population. Therefore, future FDs may be unable to acquire the historical clinical competencies.

Community pharmacy placements would allow for communication skills, medical 
14 and pain history taking, and working within a team to be tested and improved - all fundamental skills essential in the dental profession. Communication is an indispensable element for building rapport and relationships between patient and dentist, and is associated with increased efficiency, more accurate diagnosis, improved patient satisfaction and reduced likelihood of complaints or litigation. ${ }^{8}$ It is within the FD's scope of practice to diagnose disease and prescribe medications as part of dental treatment, ${ }^{9}$ allowing patients to be managed effectively and appropriately through the 'triple A' approach: advice, analgesics and antimicrobials, within a community pharmacy setting. Undoubtedly, skills and confidence around prescribing, drug mechanisms, drug interactions and writing prescriptions can be mastered while under the supervision and teaching of principal pharmacists, standing the FD in good stead for their future career as a dentist.

There is an existing push for pharmacists themselves to have a role in delivering oral health advice to patients presenting within pharmacies with dental queries; however, it is important to consider whether the dental query is within their scope of practice. Having an FD present would provide a healthcare professional who is qualified to deal with such queries to appropriately manage non-urgent dental complaints outside of the normal dental setting. This would allow those who do not routinely visit a dentist, whether due to dental anxiety, not being registered with a dentist or inability to book an appointment, to access dental advice, which could ultimately transform the oral care of many patients. Furthermore, a learning opportunity would be provided for members of the pharmacy team from the FD's ability to educate them on how to manage over-the-counter dental problems.

Oral health is fundamental for both general health and wellbeing. The concept of a close working relationship between dentists and pharmacists is not new, and this idea expands on the previous work implemented by Dental and Pharmacy teams at HEE LaSE by use of dental factsheets. This opportunity would allow for the implementation of collaborative inter-professional working on a local and easily accessible basis, with the integration of both dentists and pharmacists into primary care networks which, if successful, could be implemented nationwide.

\section{References}

1. Public Health England. National Dental Epidemiology Programme for England: Oral health survey of adults attending general dental practices 2018. 2020. Available at: https://assets.publishing.service.gov.uk/ government/uploads/system/uploads/attachment_ data/file/891208/AiP_survey_for_England_2018.pdf (accessed August 2020).

2. Public Health England. National Dental Epidemiology Programme for England: oral health survey of 5-yearolds 2019. 2020. Available at: https://assets.publishing. service.gov.uk/government/uploads/system/uploads/ attachment_data/file/873492/NDEP_for_England_OH Survey_5yr_2019_v1.0.pdf (accessed August 2020).

3. Government Office for Science. Future of an ageing population. 2016. Available at: https://www.gov. uk/government/publications/future-of-an-ageingpopulation (accessed August 2020)

4. Sturrock A, Cussons $\mathrm{H}$, Jones C etal. Oral health promotion in the community pharmacy: an evaluation of a pilot oral health promotion intervention. Br Dent J 2017; 223: 521-525.

5. Wilson N, Soni A. Interprofessional working: a spearhead opportunity for dentistry and pharmacy. $\mathrm{Br}$ Dent J 2016; 221: 607-608.

6. Health Education England London and South East Pharmacy. Dental information. Available at: https:// www.lasepharmacy.hee.nhs.uk/primary-care/dentalfactsheets/ (accessed August 2020).

7. Royal College of Surgeons of England. Dental Foundation Training (previously known as Vocational Training). Available from: https://www.rcseng.ac.uk/ dental-faculties/fds/nacpde/overseas-qualifieddentists/uk-employment/dental-foundation-training/ (accessed August 2020).

8. Waylen $A$. The importance of communication in dentistry. Dent Update 2017; 44: 774

9. General Dental Council. Scope of practice. 2019. Available at: https://www.gdc-uk.org/informationstandards-guidance/standards-and-guidance/scopeof-practice (accessed August 2020).

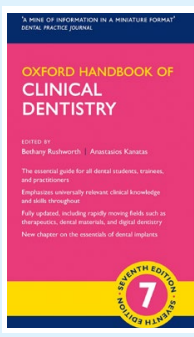

\section{OXFORD HANDBOOK OF CLINICAL DENTISTRY, 7TH EDITION}

Edited by Bethany Rushworth and Anastasios Kanatas;

2020;

Oxford University Press;

f32.99; pp. 864;

ISBN: 9780198832171

The seventh edition of the Oxford handbook of clinical dentistry offers a comprehensive overview of the fundamentals of clinical practice, providing readers with a baseline knowledge of clinical dentistry, dental specialities and more. The new edition covers a wealth of information which reflects contemporary dental practice. It is an essential read for dental undergraduates, postgraduates, hygiene and therapy students, general dental practitioners and dentist clinicians working in hospitals.

The handbook is clearly and concisely written, comprising 21 chapters. Each chapter is outlined using clear subheadings, with simple visual aids and links to relevant pages in other chapters signposted throughout. In addition, directing the learner to further reading and academic literature is an invaluable feature of the handbook, as it encourages readers to enhance their knowledge and understanding beyond the content of the handbook.

The editors cover the basics of preventative and paediatric dentistry, orthodontics, oral surgery, oral medicine and maxillofacial surgery. There are five clearly outlined chapters on restorative dentistry, which are thoroughly described and easy to read. Fundamental topics such as medicine relevant to dentistry, therapeutics, analgesia, anaesthesia, sedation and dental materials are also explained. The final chapters, including law and ethics, professionalism and communication, and practice management make for a comprehensive handbook which is incredibly useful to the intended audience. Information on passing exams, social media and risks to professionals, as well as relevant websites and links, are outlined towards the end of the book in the chapter titled 'Useful information and addresses. Conveniently, the handbook is lightweight and can be easily taken to the clinic.

Overall, the Oxford handbook of clinical dentistry covers a large scope of content, and provides a key overview of all aspects of clinical dentistry and the dental specialities. This handbook also provides appropriate revision material for those wishing to undertake undergraduate and postgraduate exams including the MJDF and MFDS. This book is of high quality, is well referenced and is affordable for students. It is a valuable handbook for any student, trainee, or qualified dentist treating patients in both a clinical and hospital setting. 